\title{
LINEAMIENTOS Y ESTRATEGIAS PARA MEJORAR LA CALIDAD DE LA ATENCIÓN EN LOS SERVICIOS DE SALUD
}

\author{
José Carlos Del Carmen Sara1,a
}

\begin{abstract}
RESUMEN
El desafío de la Cobertura Universal en Salud (CUS) orientó a los países a desplegar sus mayores esfuerzos en la ampliación de la proporción de población protegida, la delimitación y ampliación de sus planes de beneficios, así como en la provisión de recursos financieros que permitan respaldar el pago de las prestaciones brindadas. En nuestro país, a través de la implementación de la política de aseguramiento en salud, ha sido posible arribar a importantes logros, evidenciando, sin embargo, un insuficiente efecto en el acceso oportuno y en la satisfacción de las necesidades de salud de gran parte de la población. En el presente artículo partimos por destacar los principales avances y limitaciones en el proceso hacia una CUS, orientando el desarrollo de la agenda pendiente, sobre la base de los planteamientos emitidos por Organizaciones Internacionales que apuntan hacia una mejora de los sistemas de salud a nivel global. Los desafíos pendientes incluyen esfuerzos de involucramiento y articulación de los diversos actores, en la tarea de rediseñar los procesos de atención, fortalecer la dimensión ética de su ejercicio, así como promover la participación ciudadana en la generación de un sistema de salud de alta calidad, que permita un acceso efectivo y oportuno a servicios de salud; ello obliga a adoptar medidas que alcancen a todo el sistema de salud, orientadas en una visión compartida y liderada por los responsables de su conducción y gobierno.
\end{abstract}

Palabras clave: Perú; Cobertura Universal en Salud; Calidad; Cuidados en salud; Gobernanza (fuente: DeCS BIREME).

\section{GUIDELINES AND STRATEGIES TO IMPROVE THE QUALITY OF CARE IN HEALTH SERVICES}

\begin{abstract}
The challenge of Universal Health Coverage (UHC) led countries to make greater efforts to increase the proportion of population protected, to define and expand their benefit plans, and to provide financial resources to support payment for the benefits provided. The implementation of the health insurance policy in our country has allowed reaching important milestones, evidencing, however, an insufficient effect on timely access and on meeting the health needs of a large portion of the population. In this article we begin by highlighting the main advances and limitations in the process towards a UHC, guding the development of the pending agenda, based on the proposals issued by international organizations aimed at improving health systems globally. The pending challenges include efforts to involve and articulate the various actors in the task at hand of redesigning healthcare processes, strengthening the ethical dimension of their practice, as well as promoting citizen participation in the generation of a high-quality health system that would facilitate effective and timely access to health services. This requires the adoption of measures that extend to the entire health system, based on a shared vision and led by those responsible for its execution and governance
\end{abstract}

Keywords: Perú; Universal health coverage; Quality, Healthcare; Governance (source: MeSH NLM).

\section{INTRODUCCIÓN}

El desafío de la Cobertura Universal en Salud (CUS), constituye un objetivo global inscrito en las metas a alcanzar para el 2030, en el que se define a la salud como «un impulsor, un indicador y un resultado del desarrollo sostenible» (1,2). En este contexto, las decisiones gubernamentales, la formulación e implementación de políticas, la movilización de recursos, el involucramiento e impulso desde el seno de la sociedad en su conjunto, deben propugnar al logro de este objetivo, como medio y fin para el desarrollo y bienestar de nuestra población ${ }^{(3)}$; sin embargo, este logro no resultaría suficiente, si no se toma en cuenta el acceso efectivo, oportuno y de calidad a los servicios de salud requeridos. En el presente artículo partimos por destacar los principales avances y limitaciones en el proceso

\footnotetext{
Superintendencia Nacional de Salud. Lima, Perú

a Médico neurólogo, doctor en Salud Pública

Recibido: 09/04/2019 Aprobado: 22/05/2019 En línea: 28/06/2019
} 
hacia una CUS en el Perú, orientando el desarrollo de la agenda pendiente, sobre la base de los planteamientos emitidos por Organizaciones Internacionales que apuntan hacia una mejora de los sistemas de salud a nivel global.

\section{EL DESAFÍO DE LA COBERTURA UNIVERSAL EN SALUD EN EL PERÚ}

En el Perú, luego de los esfuerzos realizados por hacer realidad los objetivos consignados en la Conferencia de Alma-Atá, por parte de los estados miembros de la Organización Mundial de la Salud (OMS), que demandaba «Salud para todos en el 2000» (4), se plasmó un importante hito, en el marco del «Acuerdo Nacional» suscrito en el 2002, que da inicio al derrotero transitado por nuestro país en las últimas décadas. Dicho acuerdo, suscrito con participación, no sólo de los partidos políticos, sino también de los diferentes representantes de gremios, organizaciones sociales, religiosas y del Gobierno, consigna como Décimo Tercera Política de Estado, el «Acceso Universal a los Servicios de Salud y a la Seguridad Social», precisando que este debía darse «en forma gratuita, continua, oportuna y de calidad, con prioridad en las zonas de concentración de pobreza y en las poblaciones más vulnerables» ${ }^{(5,6)}$.

Es así que surge el Seguro Integral de Salud (SIS), sobre la base de los entonces llamados «Seguro Escolar Gratuito" y «Seguro Materno Infantil»; dicha institución para el 2004, llegaba a cubrir al $14,9 \%$ de la población, que se sumaba al $17,4 \%$ de población adscrita a ESSALUD y $5 \%$ a otros seguros de salud ${ }^{(7)}$.

En el 2009, se promulga la Ley de Aseguramiento Universal en Salud, consignando como objetivo que el $100 \%$ de la población debía contar de manera progresiva con un seguro de salud, que lo proteja frente a los costos de una posible enfermedad, lo que trajo consigo cambios significativos en la manera como se accedía y financiaba la atención en los servicios públicos de salud ${ }^{\left({ }^{8)}\right.}$. Cabe señalar, que en la década precedente, el financiamiento de las atenciones era realizado fundamentalmente por los propios pacientes, a través de tarifas establecidas en los establecimientos públicos de salud, con el fin de compensar la escases de recursos, lo que conllevó a que la mayor parte de hospitales con capacidad de «recaudar recursos», sostuviesen el financiamiento de sus posibles mejoras en estructura o equipamiento, incluyendo el pago de incentivos a sus trabajadores, generando una distorsión progresiva de la razón de ser de los servicios públicos, que derivó en desmedro de la calidad y oportunidad de la atención de la población de menores recursos, subvencionada por el SIS ${ }^{(9-13)}$.

En este contexto, frente a una política de aseguramiento no alineada a un adecuado financiamiento, se derivó en un progresivo desprestigio de un SIS incapaz de poder cumplir de manera oportuna y suficiente con las transferencias de recursos a los establecimientos de salud, trayendo como consecuencia un creciente rechazo o limitación de la atención a sus afiliados, que pasaron a poseer una cobertura sin acceso a servicios de salud.

Es a partir del 2012, en que se promueve desde el Ministerio de Salud (MINSA), un importante esfuerzo en dotar de los recursos necesarios al SIS para cumplir con el mandato que la ley le demandaba, permitiendo triplicar su asignación presupuestaria en sólo cuatro años (Figura 1), esto conllevó a que progresivamente los hospitales y pacientes fuesen identificando al SIS como una institución que respaldaba el financiamiento de la atención de las personas más pobres, aunado a la ampliación de su cobertura a atenciones de alto costo, iniciando con ello el proceso de recuperación de la razón de ser de los servicios públicos, al servicio de los más desprotegidos ${ }^{(14,15)}$.

\section{LA COBERTURA UNIVERSAL EN SALUD EFECTIVA Y EL ENFOQUE DE CALIDAD}

La concepción tridimensional del abordaje para la obtención de una CUS, planteado por la OMS en su informe anual del 2010, orientó a los países a desplegar sus mayores esfuerzos para alcanzar este objetivo, sobre la base de ampliar la cobertura de población protegida, de delimitar y ampliar progresivamente sus planes de beneficios, así como proveer los recursos financieros que respaldara la ampliación de dichas coberturas, sea a través de mayor asignación de recursos públicos, como del diseño de nuevos mecanismos de aporte, para una contribución de quienes poseían capacidad de pago ${ }^{(16)}$.

Los efectos en nuestro país, pudieron hacerse evidentes a través de las mediciones realizadas en la Encuesta Nacional de Hogares (ENAHO), que mostraron que mientras para el 2004 , el $24,7 \%$ de la población que presentaba un problema de salud, no acudía a un establecimiento de salud por "falta de dinero», para el 2017 este porcentaje se redujo a sólo un 5,6\% (7). Estos resultados, permitían constatar que los esfuerzos realizados para ampliar la cobertura poblacional, los planes de beneficios y el incremento del presupuesto asignado al SIS, devenían en un avance real hacia el cumplimiento de los objetivos planteados; sin embargo, el incremento de la demanda, en un sistema de servicios de salud fragmentado y segmentado, saco a relucir las limitaciones derivadas de estas deficiencias estructurales, conllevando a una disrupción entre la cobertura ofertada y el acceso efectivo a los servicios requeridos ${ }^{(14,15)}$. Quedaba claro que esta mirada inicial, había mantenido postergada una "cuarta dimensión», que implicaba un desafío mayor pero indispensable para que este carácter universal de acceso a la salud sea realmente efectivo, es decir, la 


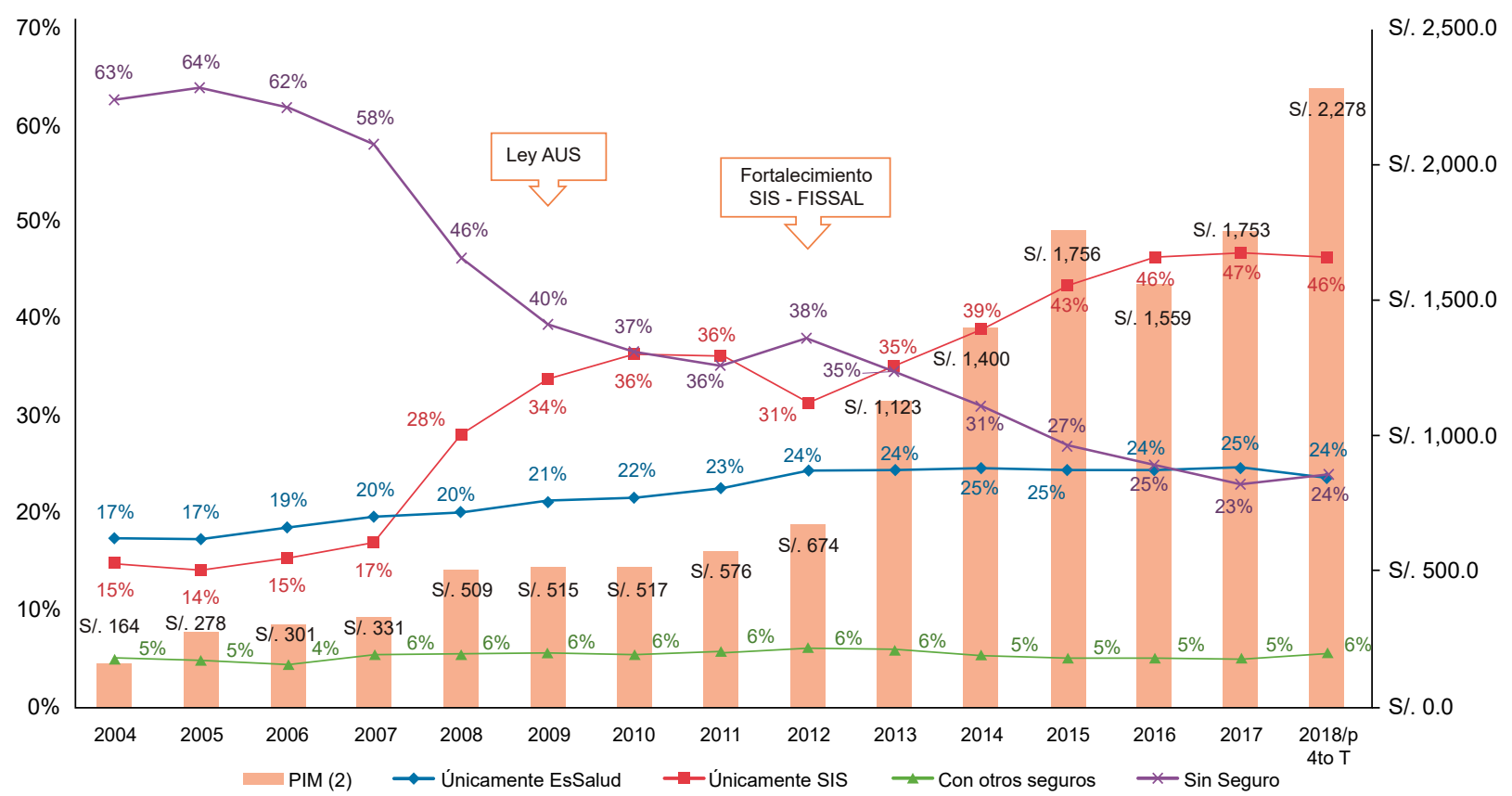

PIM: Presupuesto Institucional Modificado, EsSalud: Seguro Social, SIS: Seguro Integral de Salud, AUS: Aseguramiento Universal en Salud, FISSAL: Fondo Intangible Solidario de Salud

Fuente: Informe Técnico No 1 marzo 2019. Condiciones de Vida en el Perú octubre-diciembre 2018. INEI, Consulta amigable. Marzo 2019. Ministerio de Economía y Finanzas. Elaboración propia

Figura 1. Evolución de la cobertura de aseguramiento y financiamiento del Seguro Integral de Salud, 2004-2018

dimensión de la calidad de los servicios (Figura 2), a los que la población debería acceder de manera oportuna, efectiva y adecuada ${ }^{(16)}$.

\section{SALUD GLOBAL SOBRE LA BASE DE SISTEMAS DE SALUD DE ALTA CALIDAD}

Para el 2012, en que se evaluaba el logro de los «Objetivos de Desarrollo del Milenio» y la definición de los nuevos desafíos a plantearse para los años 2015 al 2030, se vio la necesidad de identificar y relevar la importancia del abordaje de la calidad, para un acceso efectivo a los servicios de cuidado de la salud, es así que esta fue inscrita en la redacción de los Objetivos de Desarrollo Sostenible $(\mathrm{ODS})^{(17)}$.

La Comisión Lancet de Salud Global (CLSG) sobre sistemas de alta calidad en la era de los ODS, constituida con el objeto de identificar y difundir las mejores experiencias que permitan orientar este abordaje, remarcó el hecho que «si bien en las últimas décadas los países de bajos y medianos ingresos (PBMI) han mejorado sus resultados en materia de salud", ello los enfrenta a nuevos desafíos, que responden a los "cambios en las necesidades médicas, las crecientes expectativas de los ciudadanos y los nuevos y ambiciosos objetivos del sector salud» ${ }^{(18)}$. Sin duda, los avances realizados en Perú no resultaban suficientes, demandando un esfuerzo mayor que permita cubrir las expectativas de una población mejor empoderada, de una cobertura que exige no sólo mayores recursos económicos o de infraestructura, sino de una real estructuración de un sistema de salud integrado y coordinado, con mayor dotación de recursos humanos y gestores calificados y comprometidos en el logro de una mejor atención al servicio de los ciudadanos.

En esta misma línea, la Organización de Cooperación para el Desarrollo Económico (OCDE), en su informe sobre el desempeño del Sistema de Salud del Perú al 2017, señala que para poder garantizar un avance sostenido en este desafío de alcanzar una CUS, con calidad en el acceso, es preciso generar mecanismos que permitan evaluar y monitorear las metas de política, con «datos relevantes, oportunos y precisos a lo largo de todo el sector salud y del país», lo que implica el desarrollo de un sistema de información de salud «que atienda las necesidades del análisis nacional y de formulación de políticas para un desempeño mejorado del sistema» (13). Es así que el despliegue futuro de estrategias que permitan consolidar el avance en la implementación de esta importante política pública, nos exige enfrentar debilidades estructurales y de procesos en nuestro sistema prestacional, acompañado de una sistematización de información relevante, que oriente la toma de decisiones sobre la base de evidencias sólidas, 


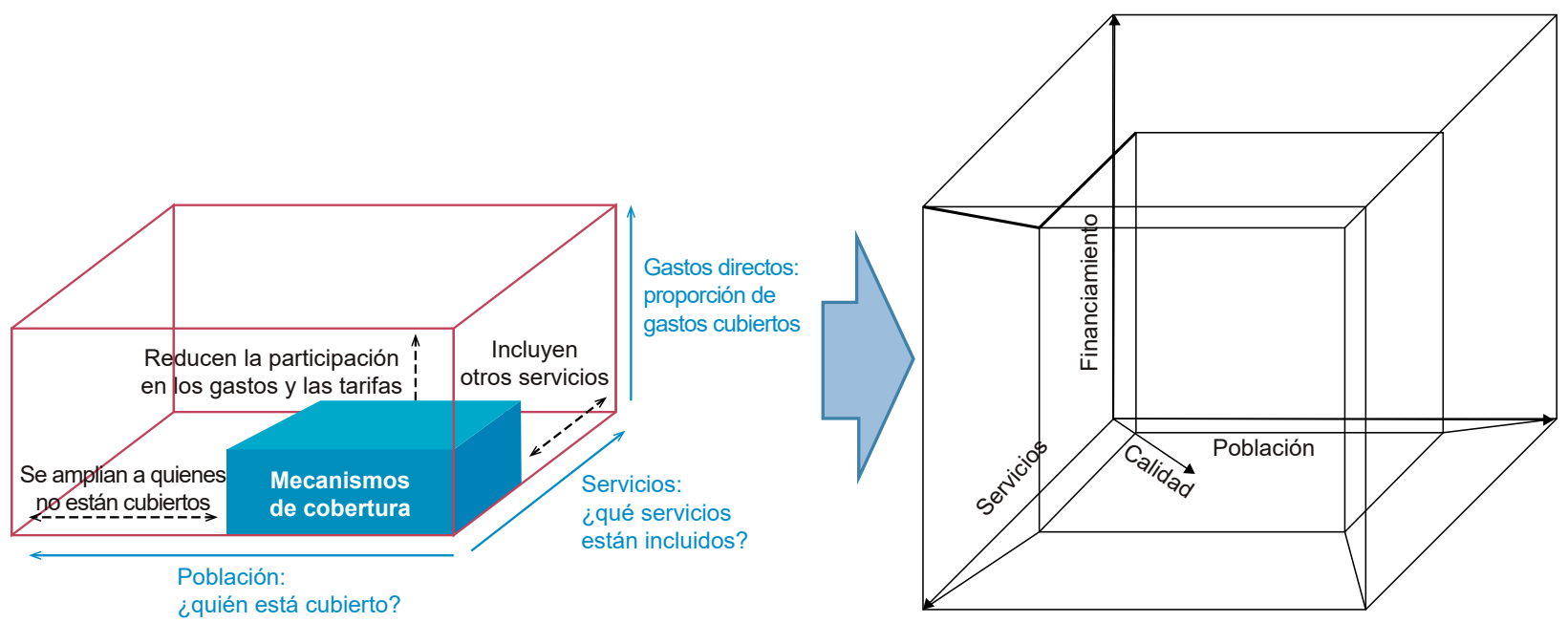

Tomado de Organización Mundial de la Salud (OMS). Informe sobre la salud en el mundo 2010. Adaptación propia.

Figura 2. Enfoque de Calidad para una efectiva Cobertura Universal en Salud

para una mejora del desempeño y resultados en salud, que pueda ser percibido y redunde en beneficio de los ciudadanos $^{(19)}$.

\section{RECOMENDACIONES PARA EL DISEÑO DE ESTRATEGIAS DE MEJORA DE LA CALIDAD CON RECURSOS LIMITADOS}

De la evaluación realizada por la CLSG, se consideró necesario relevar la importancia de las «dimensiones éticas de los cuidados de alta calidad en entornos con recursos limitados» (18)

El visibilizar la dimensión ética del desafío por alcanzar la CUS, confronta a los países a sincerar su verdadera voluntad por impulsar este proceso a pesar de la limitación de sus recursos, obligando a priorizar con criterios de equidad, la identificación y canalización de los recursos necesarios para su efectiva y sostenible implementación; de este planteamiento se derivaron cinco conclusiones, que la comisión considera deben tomarse en cuenta en el diseño de estrategias de mejoramiento del acceso y calidad de los servicios de salud, en países de bajos y medianos ingresos ${ }^{(18)}$. Estas parten por reconocer que «los cuidados que recibe la población suelen ser inadecuados y de baja calidad asistencial, afectando en mayor grado a los grupos más vulnerables», hecho señalado también por la OCDE, en su informe sobre el desempeño del Sistema de Salud del Perú al 2017, destacando que si bien se ha llegado a mejorar el acceso y la protección financiera, aun nuestra población "continúa experimentando tasas altas de enfermedades infecciosas, junto con una prevalencia creciente de enfermedades no transmisibles», que implica un mayor esfuerzo, en particular para llegar a la población de menores recursos ${ }^{(13)}$. Así mismo se destaca, como «los sistemas de salud de alta calidad pueden salvar más de 8 millones de vidas al año en los países de bajos y medianos ingresos», desafío que se traduce en nuestro país en revertir las graves inequidades en acceso y resultados de salud, que afecta a la población de menores recursos, generando nuevas estrategias que permitan brindar una mejor ruta de acceso a una atención integral y continua, en los diferentes niveles de atención, articulando e implementando mecanismos y arreglos institucionales que lo hagan posible ${ }^{(18,20)}$.

La importancia de la medición de resultados y de una adecuada comunicación en salud también es relevada, al señalar que «los sistemas sanitarios deben medir $y$ comunicar aquello que más les importa a los ciudadanos»; ello implica hacer visible los logros y mejoras en los mejores prestadores, impulsando los procesos de acreditación y reconocimiento a dichos establecimientos, función que fue asignada a la Superintendencia Nacional de Salud (SUSALUD) en el 2013, pero luego retornada al MINSA tres años después, a la par que se decidió retirar la Dirección de Calidad de la estructura orgánica del ministerio, debilitando una función que estaba inscrita en la Política de Gestión de la Calidad en Salud, impulsada desde el $2002^{(21,22) .}$

Las dos últimas conclusiones de la CLSG relevan la especial importancia de la Investigación «para lograr transformar los sistemas sanitarios de baja calidad en sistemas de alta calidad», así como el hecho de que una «mejora de la calidad asistencial exige tomar medidas en 
todo el sistema de salud». Ello conlleva para nuestro país, el abordar debilidades estructurales, relacionadas con la fragmentación de la oferta de servicios de salud, la diversidad y no alineamiento de las fuentes de financiamiento, la escasez de recursos humanos especializados, tanto en la prestación como en la gestión de los limitados recursos disponibles, entre otros, que permita concretar los procesos de articulación, coordinación e integración del sistema ${ }^{(23) .}$

\section{EL ENFOQUE DE CALIDAD COMO MEDIO Y FIN PARA UNA MEJORA EN EL SISTEMA DE SALUD}

La necesidad de incorporar el enfoque de calidad, como eje orientador para la mejora de los sistemas de salud en PBMI, así como la adopción de medidas que lleguen a alcanzar a todo el sistema, implica para la CLSG, el desarrollo de cuatro acciones fundamentales ${ }^{(18)}$ :

\section{LIDERAZGO EN LA GESTIÓN}

"Los directivos de los sistemas sanitarios deben regirse por la búsqueda de la calidad». El involucramiento de las autoridades sanitarias y no sólo de los equipos técnicos, es indispensable para construir y sostener una «visión compartida de la calidad asistencial», ello implica promover el compromiso y liderazgo de los responsables de la gestión de los servicios, así como de las autoridades políticas responsables en el ámbito subnacional. Instituciones como SUSALUD, han venido incorporando un especial énfasis en acciones que buscan prevenir los riesgos que conllevan a una mala atención, identificándolos conjuntamente con los responsables de la gestión sanitaria, con el objeto de instaurar planes de mejora que eviten la aplicación de multas y coadyuven a la mejora de sus servicios, en beneficio de los ciudadanos ${ }^{(24)}$. El involucramiento del sector privado, la articulación de los diferentes prestadores públicos y de la seguridad social, así como la participación de la sociedad civil, a través de las «Juntas de Usuarios en Salud» (JUS), forma parte de las estrategias que vienen siendo impulsadas por dicha entidad, para el abordaje sistémico que conlleve a una mejora de la calidad prestacional ${ }^{(13)}$.

\section{REDISEÑO DE PROCESOS}

«Los países deben rediseñar la prestación de servicios para optimizar los resultados sanitarios en lugar de centrarse únicamente en el acceso geográfico a los servicios». En Perú se ha realizado un importante esfuerzo en las tres últimas décadas, con el fin de aproximar los servicios de salud hacia la mayor parte de la población; sin embargo, la dispersión y escasa planificación en esta tarea, derivó en una débil presencia, con escasa capacidad resolutiva, ocasionando poca credibilidad en la utilidad de este primer contacto, conllevando a abarrotar los hospitales con una demanda que bien podría ser resuelta en un primer nivel de atención. Recuperar o construir un lazo de confianza, con esa puerta de entrada al sistema de servicios de salud, implica rediseñar el modelo, romper las barreras entre los diferentes subsectores, generar mecanismos de interoperabilidad, que permitan constituir verdaderas Redes Integradas de Servicios de Salud, que brinden una atención primaria concebida con criterios de integralidad y continuidad en el proceso, en los diferentes niveles de atención ${ }^{(25)}$.

\section{PERSONAL IDÓNEO}

«Los países deben transformar la fuerza laboral sanitaria basada en idoneidad, apoyando y respetando mejor a todos los empleados para que presten los mejores cuidados posibles». El desafío de la dotación y distribución de Recursos Humanos en Salud, fue identificado por la OMS, como un factor indispensable para el fortalecimiento de los sistemas de salud en los diversos países, por lo que denominó a la década 2005 al 2015 como la «Década de los Recursos Humanos en Salud», realizando un "llamado a la acción" para desarrollar e implementar políticas que permitan identificar y abordar los diferentes componentes que conllevan a esta situación (26). En Perú, se desarrollaron algunas investigaciones con el objeto de sistematizar y dar seguimiento a dichos componentes, desde el campo de la formación de Recursos Humanos en Salud, hasta las distorsiones y limitaciones existentes en el campo del trabajo, de la gestión descentralizada y de las débiles políticas, que permitiesen promover un mejor desempeño con condiciones de un real desarrollo humano de los profesionales y trabajadores del sector salud. Estos desafíos, conllevaron importantes esfuerzos, que buscaron entre otros, disminuir las brechas existentes en las remuneraciones de los profesionales que laboraban en hospitales de mayor demanda y complejidad, que podían otorgar «incentivos laborales» originados en base a la «recaudación» de dichos establecimientos, condición que no se daba en los pequeños establecimientos de salud, constituyendo un incentivo inverso a las necesidades que el abordaje de la inequidad sanitaria nos demandaba ${ }^{(27)}$, esta distorsión debilitó en algunos casos, el espíritu y entrega que caracterizaba a los profesionales del sector, al sentirse injustamente postergados. Las tensiones derivadas de la defensa de los derechos laborales, la mayor demanda de la población, el progresivo empoderamiento ciudadano, hacen visible la necesidad de abordar y fortalecer la formación «ética» en los profesionales y trabajadores del sector, que promueva un trato respetuoso y responsable a los usuarios de los servicios de salud, pero a su vez la tarea de educar a la ciudadanía, en el respeto a los profesionales y trabajadores que los sirven; así mismo, resulta indispensable el establecer mecanismos de reconocimiento y de incentivos por parte de la instituciones de las cuales ellos dependen.

\section{PARTICIPACIÓN CIUDADANA}

«Los gobiernos y la sociedad civil deben transmitir a la población la importancia de demandar calidad, para 
así empoderarlos a exigir esta responsabilidad a los sistemas y a buscar activamente cuidados de alta calidad». El lograr visibilizar esta «cuarta dimensión» del desafío de lograr una CUS referida a la Calidad, nos obliga a identificar y fortalecer los mecanismos y espacios gubernamentales y no gubernamentales que puedan contribuir en impulsar las acciones necesarias desde la ciudadanía, así como desde los diferentes actores del sistema de salud en nuestro país ${ }^{(16,28)}$. En este contexto, el rol de la SUSALUD podría resultar fundamental, como parte de las estrategias a desplegar en la mejora de los servicios de salud en el Perú ${ }^{(13)}$.

\section{EL ROL DE LA SUSALUD EN LA MEJORA DE LOS SERVICIOS DE SALUD}

SUSALUD, inició sus funciones en el Perú en el 2013, sobre la base de la Superintendencia Nacional de Aseguramiento en Salud (SUNASA), creada a su vez en el 2009, a través de la Ley Marco de Aseguramiento Universal en Salud, que extendió los alcances de la entonces Superintendencia de Entidades Prestadoras de Salud (SEPS), encargada de «autorizar, regular y supervisar» el funcionamiento de las entidades privadas que administraban fondos de aseguramiento en salud para los trabajadores que optaban por dicho sistema, en el marco de la aplicación de la Ley de Modernización de la Seguridad Social en Salud, promulgada en 1997. La evolución y alcances del rol de las entidades que culminaron en el inicio de funciones de SUSALUD (Figura 3), reflejan los diferentes procesos mediante los cuales nuestro país buscaba ampliar la cobertura y acceso a la salud de los peruanos, enfocado en la década del 90, en la participación del sector privado en la «modernización de la seguridad social», dando a la SEPS la función de cautelar el adecuado funcionamiento de los aseguradores y prestadores privados de servicios de salud, que tenían a cargo a alrededor de un millón de peruanos; la ampliación de tal alcance hacia todos los asegurados, asignado a SUNASA, reconocía la necesidad de dotar a más de 20 millones de afiliados al SIS o ESSALUD, de contar también con una entidad que proteja la adecuada protección y atención de su salud ${ }^{(29,30)}$.

El rol asignado a SUSALUD, incluye en sus funciones la «Promoción, protección y defensa de los derechos de las personas al acceso a los servicios de salud, supervisando que las prestaciones sean otorgadas con calidad, oportunidad, disponibilidad y aceptabilidad, con independencia de quien las financie», alcanzando esta cautela de derechos a más de 30 millones de peruanos, lo que constituye un desafío y una oportunidad, para impulsar estrategias que conlleven a una mejora de la calidad de los servicios otorgados, en el marco de la política de ampliar la cobertura de protección en salud, a través del aseguramiento universal en salud en el Perú ${ }^{(21,30)}$.

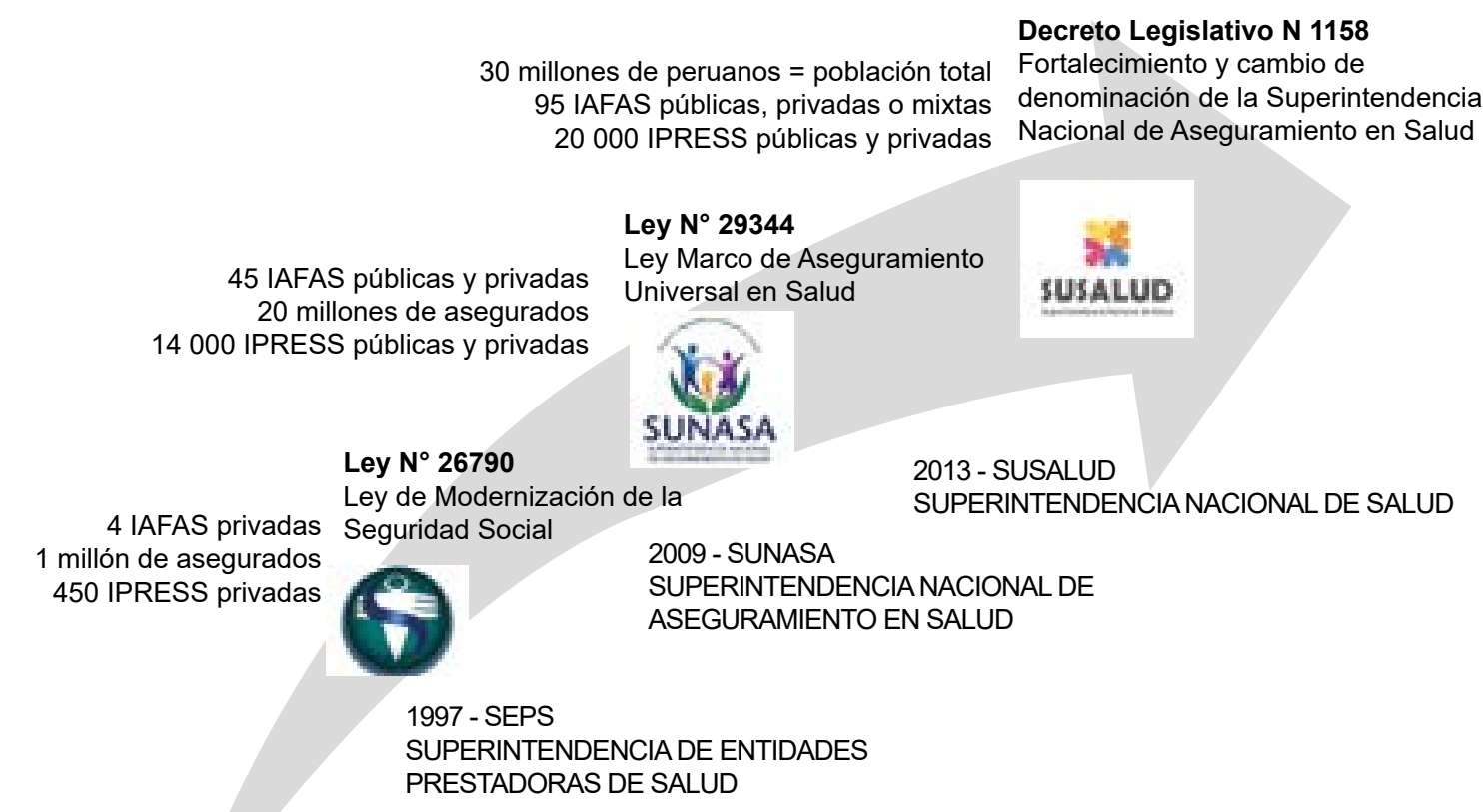

IAFAS: Institución Administradora de Fondos de Aseguramiento en Salud, IPRESS: Instituciones Prestadoras de Servicios de salud Fuente: Elaboración propia

Figura 3. Evolución y alcances de las entidades encargadas de la regulación y supervisión del financiamiento y prestación de salud en el Perú 
En este contexto, el fortalecimiento institucional y el desarrollo de nuevas estrategias por parte de SUSALUD, para la mejora de la «calidad, oportunidad, disponibilidad y aceptabilidad" de las prestaciones de salud, se constituye en una necesidad para impulsar este proceso. Adicional a ello, la OCDE destaca en su informe al 2017, los avances y limitaciones que afronta SUSALUD, señalando que «actualmente no tiene alcance regional y tampoco los recursos financieros, pero la capitalización de sus bien establecidas prácticas y de la buena relación con las partes interesadas, podría ser una forma de impulsar una gestión más sólida de datos y normas»; es decir, releva la potencialidad e importancia de esta institución, incluyendo su función de recopilar, analizar, transferir, difundir e intercambiar la información generada por los diferentes actores del sistema de salud, así como la medición de la experiencia de los usuarios durante la prestación de dichos servicios ${ }^{(13)}$.

En la búsqueda del logro de estos objetivos, recientemente SUSALUD ha determinado la reorientación de sus intervenciones, en el marco de una nueva Política Institucional que prioriza la «prevención de la vulneración de los derechos en salud», a través de la identificación y «Gestión del Riesgo», promoviendo el desarrollo e implementación de «Planes de Mitigación», que conlleven a una mejora en la calidad de los servicios; para ello viene impulsando la firma de Convenios con Gobiernos Regionales, que permita involucrarlos en esta tarea, con un enfoque territorial, que integre a todos los actores del sistema; es decir, se ha transitado de un accionar reactivo que sanciona infracciones, hacia un accionar enfocado en prevenirlas y corregirlas, con el objeto de obtener como resultado una progresiva mejora en la calidad de los servicios de salud, cautelando así los derechos en salud de los ciudadanos.

\section{CONCLUSIONES}

El desafío de la CUS debe conllevar a un acceso efectivo y oportuno a servicios de salud de calidad, ello obliga a adoptar medidas que alcancen a todo el sistema de salud, orientada en una visión compartida y liderada por los responsables de su conducción y gobierno.

Los avances de nuestro país en términos de cobertura, a través de la implementación de la política de aseguramiento en salud, han traído consigo importantes logros, evidenciando, sin embargo, un insuficiente efecto en el acceso oportuno y en la satisfacción de las necesidades de salud de gran parte de nuestra población.

Los desafíos pendientes incluyen esfuerzos de involucramiento y articulación de los diversos actores, en la tarea de rediseñar los procesos de atención, fortalecer la dimensión ética de su ejercicio, así como promover la participación ciudadana en la generación de un sistema de salud de alta calidad.

SUSALUD viene contribuyendo en este proceso, al haber reorientado los esfuerzos destinados a solo sancionar incumplimientos de los actores del sistema, priorizando más bien su involucramiento en la identificación de riesgos, el diseño e implementación de Planes de Mitigación, que conlleven a una mejora en las condiciones de la prestación, evitando así la vulneración de los derechos en salud de los ciudadanos.

La CLSG concluye su evaluación señalando que «los países sabrán que están en camino de conseguir un sistema sanitario responsable y de alta calidad cuando los profesionales de la salud y los legisladores políticos opten por recibir su atención médica en sus propias instituciones públicas»; esta sentencia nos da una pista para identificar el momento en que nos aproximemos al cumplimiento de este objetivo.

Fuentes de financiamiento: Autofinanciamiento.

Conflictos de interés: Superintendente Nacional de Salud, exjefe del Seguro Integral de Salud, ex Viceministro de Salud

\section{REFERENCIAS BIBLIOGRÁFICAS}

1. Resolution adopted by the General Assembly on 25 September 2015. Transforming our world: the 2030 Agenda for Sustainable Development. United Nations Official Document. 2015 oct [Internet]. [citado $28 \mathrm{de}$ enero de 2019]. Disponible en: http:// www.un.org/ga/search/view_doc. asp?symbol=A/RES/70/1\&Lang=E

2. Caribe CE para AL y el. La Agenda 2030 y los Objetivos de Desarrollo Sostenible: una oportunidad para América Latina y el Caribe. Objetivos, metas e indicadores mundiales [Internet]. CEPAL; 2019

3. WHO $\mid$ Strengthening health systems for universal health coverage and sustainable development [Internet]. WHO. [citado 29 de marzo de 2019]. Disponible en: http://www.who.int/bulletin/volumes/95/7/16-187476/en/

4. Rivero T de, A D. La historia de la Conferencia de Alma Ata. Rev Peru Ginecol Obstet. 2018; 64(3):361-6.
5. Nepo-Linares E, Velásquez A. El Acuerdo Nacional como espacio de consenso para la definición de los objetivos de la reforma de salud y establecer políticas de salud en el Perú. Rev Peru Med Exp Salud Pública. 2016; 33(3):540-5.

6. Acuerdo Nacional Unidos para Crecer. Acta de Suscripción del Acuerdo Nacional - 22 de julio del 2002 [Internet]. Acuerdo Nacional Unidos para Crecer: Lima; 2014 [citado 1 de abril de 2019]. Disponible 
en: https://acuerdonacional.pe/politicasde-estado-del-acuerdo-nacional/actadesuscripcion-del-an-22-de-julio-del-2002/

7. Instituto Nacional de Estadística e Informática. Condiciones de vida en el Perú. Trimestre: Octubre - Noviembre Diciembre 2018: Informe Técnico No 1. 2019. Lima: INEI; 2019.

8. Perú. Congreso de la República. Ley 29344. Ley Marco del Aseguramiento Universal en Salud. (8 de abril de 2009).

9. Sánchez-Moreno F. El sistema nacional de salud en el Perú. Rev Peru Med Exp Salud Pública. 2014; 31(4):747-53.

10. Seinfeld J, Besich N. Universal Health Coverage Assessment Peru. Glob Netw Health Equity GNHE; 2014;14.

11. Solís R, Sánchez SE, Carrión M, Samatelo $\mathrm{E}$, Rodríguez-Zubiate $\mathrm{R}$, Rodríguez M. Cobertura de seguros de salud en relación con el uso de servicios médicos, condiciones de vida y percepción de la salud en Lima, Perú. Rev Peru Med Exp Salud Pública. 2009; 26(2):136-44.

12. Velásquez A, Suarez D, Nepo-Linares E. Reforma del sector salud en el Perú: derecho, gobernanza, cobertura universal y respuesta contra riesgos sanitarios. Rev Peru Med Exp Salud Pública. 2016; 33(3):546-55.

13. Organisation for Economic Co-operation and Development - OECD. Reviews of Health Systems: Peru 2017 [Internet]. [citado 12 de octubre de 2018]. Disponible en: http://www.oecd.org/countries/peru/ oecd-reviews-of-health-systems-peru2017-9789264282735-en.htm

14. Pavone MP. El complejo proceso de la inclusión: reforma y seguro integral de salud. Lima: Universidad Peruana Cayetano Heredia, Fondo Editorial; 2018. 141 p.

15. Gutiérrez C, Romaní Romaní F, Wong P, Del Carmen Sara J. Brecha entre cobertura poblacional y prestacional en salud: un reto para la reforma de salud en el Perú. An Fac Med. 2018;79(1):65-70.
16. Organización Mundial de la Salud. Informe sobre la salud en el mundo 2010 [Internet]. Ginebra: WHO; 2010 [citado el 1 de abril de 2019]. Disponible en: https://www.who.int/whr/2010/es/

17. Organización Panamericana de la Salud - OPS. Organización Mundial de la Salud - OMS. Oficina Regional pata las Américas. 66.o Sesión del Comité Regional de la OMS para las Américas. Estrategia para el Acceso Universal a la Salud y la Cobertura Universal de Salud [Internet]. Washington DC. OMS; 2016.

18. Kruk ME, Gage AD, Arsenault C, Jordan $\mathrm{K}$, Leslie HH, Roder-DeWan S, et al. The Lancet Global Health Commission 2018 Nov; 6(11): e1196-e1252. Disponible en: http://www.thelancet.com/lancetgh

19. Flórez CEP, Chapman E, Panisset U, Arredondo A, Fitzgerald J, Reveiz L. Disponibilidad de indicadores para el seguimiento del alcance de la "Salud Universal" en América Latina y el Caribe. Rev. Panam Salud Pública. 2016; 39:330-40.

20. Guanais, Frederico C, Regalia Ferdinando, Perez-Cuevas Ricardo, Anaya Milagros, editores. Desde el paciente: Experiencias de la atención primaria de salud en América Latina y el Caribe [Internet]. Washignton DC: BID; 2018 [citado el 29 de enero de 2019]. Disponible en: https:// cloud.mail.iadb.org/desde-el-paciente

21. Decreto Legislativo 1289. Decreto Legislativo que dicta disposiciones destinadas a optimizar el funcionamiento y los servicios de la Superintendencia Nacional de Salud (28 de diciembre de 2017)

22. Decreto Supremo 008-2017-SA. Decreto Supremo que aprueba el Reglamento de Organización y Funciones del Ministerio de Salud (03 de marzo de 2017).

23. Informe final comisión de protección social [Internet]. Scribd. [citado 3 de abril de 2019]. Disponible en: https://www. scribd.com/document/364322197/ INFORME-FINAL-COMISION-DEPROTECCION-SOCIAL

24. Superintendencia Nacional de Salud SUSALUD. Plan Estratégico Institucional 2019 - 2021. Resolución de Superintendencia $\mathrm{N}^{\circ}$ 158-2018-SUSALUD/S dic 26, 2018.

25. Pan American Health Organization. Regional Office. World Health Organization. Integrated Health Services Delivery Networks. Concepts, Policy options and a road map for implementation in the Americas. Washington DC:PAHO;2011.

26. Organización Panamericana de la Salud. Organización Mundial de la Salud. $54^{\circ}$ Consejo directivo. 67a Sesión del Comité regional de la OMS para las Américas. Metas regionales en materia de recursos humanos para la salud 2007-2015. Washington, D.C., EUA; 2015 oct.

27. Arroyo J. El Proceso del Sector Salud 1990 - 1995: Colapso Operativo y Reforma Sanitaria Silenciosa. An Fac Med. 1997; 57(3):188-95.

28. Sara M. Cano-Sierra, Álvaro Giraldo, Constanza. Concepto de calidad en salud: resultado de las experiencias de la atención, Medellín, Colombia. Rev. Fac Nac Salud Pública. 2016; 34(1).

29. Perú. Congreso de la República. Ley 26790. Ley de Modernización de la Seguridad Social en Salud. (15 de mayo de 1997).

30. Decreto Supremo $N^{\circ}$ 020-2014-SA. Decreto Supremo que aprueba el Texto Único Ordenado de la Ley $\mathrm{N}^{\circ} 29344$, Ley Marco de Aseguramiento Universal en Salud (12 de julio de 2014).

Correspondencia: José Carlos Del Carmen Sara Dirección: Calle el Visitador 250, Dpto. 302. La Molina

Teléf:: (511) 997375872

Correo electrónico: jdelcarmens@gmail.com 